\title{
Molecular detection of Enterocytozoon bieneusi in farm-raised pigs in Hainan Province, China: infection rates, genotype distributions, and zoonotic potential
}

\author{
Huan-Huan Zhou ${ }^{1,2,3, a}$, Xin-Li Zheng ${ }^{4, a}$, Tian-Ming Ma ${ }^{1,2,3}$, Meng Qi ${ }^{5}$, Jing-Guo Zhou ${ }^{1,2,3}$, Hai-Ju Liu ${ }^{1,2,3}$, \\ Gang $\mathrm{Lu}^{1,2,3, *}$, and Wei Zhao ${ }^{1,2,3,6, *}$ \\ ${ }^{1}$ Department of Pathogenic Biology, Hainan Medical University, Haikou, 571199 Hainan, PR China \\ ${ }^{2}$ Hainan Medical University-The University of Hong Kong Joint Laboratory of Tropical Infectious Diseases, Hainan Medical University, \\ Haikou, 571199 Hainan, PR China \\ ${ }^{3}$ Key Laboratory of Tropical Translational Medicine of the Ministry of Education, Hainan Medical University, 571199 Haikou, PR China \\ ${ }^{4}$ College of Animal Sciences, Tarim University, Alar, 843300 Xinjiang, PR China \\ ${ }^{5}$ Institute of Animal Science and Veterinary Medicine, Hainan Academy of Agricultural Sciences, 571100 Haikou, PR China \\ ${ }^{6}$ Department of Parasitology, Wenzhou Medical University, Wenzhou, 325035 Zhejiang Province, PR China
}

Received 1 November 2019, Accepted 19 February 2020, Published online 4 March 2020

\begin{abstract}
Enterocytozoon bieneusi is a zoonotic fungal pathogen with a high degree of host diversity that can parasitize many animals, including humans. Pigs may play an important role in the epidemiology of E. bieneusi as reservoir hosts. Nevertheless, the genotypes of E. bieneusi in pigs in China remain poorly understood. The aim of this study was to determine the prevalence of $E$. bieneusi infection amongst pigs raised on farms from four cities of Hainan Province, using nested polymerase chain reaction (PCR) of the partial small subunit of the ribosomal RNA gene, and to identify genotypes of E. bieneusi isolates based on sequence analysis of the ribosomal internal transcribed spacer (ITS) region. Among 188 stool samples, E. bieneusi was detected in 46.8\% (88/188). Eight genotypes including four known (EbpA, CS-4, MJ14, and CHG19) and four novel (HNP-I - HNP-IV) genotypes were identified. Using phylogenetic analysis, genotypes EbpA, CS4, CHG19, HNP-III, and HNP-IV were clustered into zoonotic Group 1, while the remaining three genotypes (MJ14, HNP-I, and HNP-II) clustered into Group 10. The high prevalence of zoonotic genotypes of E. bieneusi among pigs suggests that pig farming is a potential source of human infection. Additionally, this is the first identification of genotypes in Group 10 in pigs indicating unique epidemic features of E. bieneusi in pigs in Hainan Province, the southernmost part of China.
\end{abstract}

Key words: Enterocytozoon bieneusi, Genotype, ITS region, Pigs.

Résumé - Détection moléculaire d'Enterocytozoon bieneusi chez les porcs d'élevage dans la province de Hainan en Chine : taux d'infection, répartition des génotypes et potentiel zoonotique. Enterocytozoon bieneusi est un pathogène fongique zoonotique avec une grande diversité d'hôte qui peut parasiter de nombreux animaux, y compris les humains. Les porcs peuvent jouer un rôle important dans l'épidémiologie d'E. bieneusi en tant qu'hôtes réservoirs. Néanmoins, les génotypes d'E. bieneusi chez le porc en Chine restent mal connus. Le but de cette étude était de déterminer la prévalence de l'infection par E. bieneusi chez les porcs élevés dans des fermes de quatre villes de la province de Hainan, en utilisant la réaction en chaîne par polymérase emboîtée (PCR) de la petite sous-unité partielle du gène de l'ARN ribosomal et de identifier les génotypes des isolats d'E. bieneusi sur la base d'une analyse de séquence de la région des espaceurs internes transcrits ribosomiques (ITS). Sur 188 échantillons de selles, E. bieneusi a été détecté dans 46,8 \% (88/188). Huit génotypes, dont quatre génotypes connus (EbpA, CS-4, MJ14 et CHG19) et quatre génotypes nouveaux (HNP-I à IV), ont été identifiés. Dans une analyse phylogénétique, les génotypes EbpA, CS4, CHG19, HNP-III et HNP-IV étaient regroupés dans le groupe zoonotique 1, tandis que les trois génotypes restants (MJ14, HNP-I et HNP-II) étaient regroupés dans le groupe 10. La prévalence élevée des génotypes zoonotiques d'E. bieneusi chez les porcs suggère que l'élevage porcin est une source potentielle d'infection humaine. De plus, il s'agit de la première identification de génotypes du groupe 10 chez les porcs, indiquant des caractéristiques épidémiques uniques d'E. bieneusi chez les porcs dans la province de Hainan, la partie la plus méridionale de la Chine.

*Corresponding authors: luganghn@163. com; hayidazhaowei@163.com

${ }^{\text {a }}$ These authors contributed equally to this work.

This is an Open Access article distributed under the terms of the Creative Commons Attribution License (https://creativecommons.org/licenses/by/4.0), which permits unrestricted use, distribution, and reproduction in any medium, provided the original work is properly cited. 


\section{Introduction}

Microsporidia are a diverse group of obligate intracellular eukaryotic fungi pathogens. They are comprised of approximately 200 genera and 1500 species. To date, 17 species of microsporidia have been recognized as emerging human pathogens [23]. Among them, Enterocytozoon bieneusi is the most common species detected both in healthy and immunocompromized individuals that can cause increased mortality through life-threatening diarrhea, particularly in acquired immunodeficiency syndrome (AIDS) patients, children, and transplant recipients [27]. Enterocytozoon bieneusi has a high degree of host diversity and can parasitize almost all animal phyla [41]. Thus any animal can act as a potential reservoir host and contribute to environmental pollution and the continuous transmission of the disease [9]. An important step is to adequately control cross-species transmission of E. bieneusi by tracing the sources of contamination and elucidating transmission routes. However, the contribution of each animal source to human infections is poorly understood.

Sequence analyses of the internal transcribed spacer (ITS) regions of the rRNA gene have been widely applied for the detection of E. bieneusi, and this method is becoming the standard tool for E. bieneusi typing [40]. To date, ITS genotyping has contributed to the identification of over 500 genotypes, with 142 genotypes found in humans and 49 genotypes identified both in humans and animals [10, 20, 23, 31, 50, 55]. These recognized genotypes have been divided into 11 phylogenetic groups (Groups 1-11) for phylogenetic analysis [23]. To date, $132(93.0 \%)$ out of the 142 human pathogenic genotypes and $95.9 \%$ (47/49) of the zoonotic genotypes belong to Group 1 or Group 2, highlighting the genotypes with public health significance and the nature of cross-species transmission $[10,20,23,31,50,55]$. However, genotypes in Groups 3-11 appear more commonly subject to host adaptation [23]. The contribution of each animal source to human infections can be clarified by the genotyping of E. bieneusi in different animals.

Pigs are one of the most important reservoir hosts for E. bieneusi. Currently, more than 30 studies on E. bieneusi in pigs have been published from 14 countries, and 134 ITS genotypes of E. bieneusi have been identified in pigs or wild boars worldwide (Table 1). Among them, 19 genotypes (CHN1, Bfrmr2, CAF1, CS-1, CS-4, D, EbpA, EbpC, EbpD, H, Henan-III, Henan-IV, I, LW1, O, PigEBITS5, PigEBITS7, PigEB10, SH8) have been identified in humans. All genotypes found in the pigs belong to Group $1(94.8 \%, 127 / 134)$ or Group $2(5.2 \%, 7 / 134)$, suggesting that pigs play an important role in the epidemiology of E. bieneusi as a reservoir host (data based on Ref. [23]). However, the genotypes of E. bieneusi in pigs in China are not fully understood and there is a lack of data in many provinces, including Hainan Province (the southernmost part of the country).

In China, the pig industry is a major economic component in which humans and pigs live in crowded conditions, and the disease can be easily spread with a potentially major impact on the economy [57]. Hainan Province is a relatively isolated island, where E. bieneusi is prevalent in multiple animals including farmed and wild animals [60, 61]; moreover, it has been found in humans with diarrhea (unpublished data).
The pig industry represents an important poverty alleviation project in Hainan Province. However, information on the prevalence and genetic characteristics of E. bieneusi in pigs in this province is lacking. The aims of this study were to determine the prevalence and genotype distribution of E. bieneusi in pigs from four cities of Hainan Province, to provide useful information to assess the risk of zoonotic transmission.

\section{Materials and methods \\ Ethics statement}

Before beginning work on the present study, we contacted the farm owners and obtained their permission to have their animals involved. Written informed consent was obtained from the owners for the participation of their animals in this study. The protocol was also reviewed and approved by the Ethics Committee of Hainan Medical University.

\section{Collection of fecal specimens}

From March to June 2019, a total of 188 fresh fecal samples were collected from four pig farms in four cities of Hainan Province, including Baisha $(n=30)$, Danzhou $(n=58)$, Haikou $(n=20)$, and Lingshui $(n=80)$ (Fig. 1, Table 2). Farms were selected based on the owners' willingness to participate and the accessibility of animals for sampling. The number of collected specimens accounted for $20-30 \%$ of total pigs on each farm. All fecal specimens were collected from the ground immediately after defecation using a sterile disposable latex glove and placed in individual labeled sterile tubes. The ages of the pigs sampled in this study belonged to two groups: one group containing 61 pre-weaned and post-weaned pigs aged $\leq$ 60 days, and the other group containing 127 fattening pigs aged more than 60 days. At the time of sampling, all pigs were in good health. All bags were transported to our laboratory in a cooler with ice packs within $24 \mathrm{~h}$, and stored at $4{ }^{\circ} \mathrm{C}$ until processing (within $48 \mathrm{~h}$ ).

\section{DNA extraction}

All fecal specimens of the pigs were collected and sieved through an $8.0-\mathrm{cm}$-diameter sieve with a pore size of $45 \mu \mathrm{m}$. Filtrates were concentrated by centrifugation at $1500 \times g$ for $10 \mathrm{~min}$. Approximately $200 \mathrm{mg}$ of each processed sample was homogenized in $1400 \mu \mathrm{L}$ of DNA extraction buffer ASL. Genomic DNA of each specimen was extracted using a QIAamp DNA stool mini kit (QIAgen, Hilden, Germany), according to the manufacturer's recommendations. Extracted DNA was used for PCR analysis. All samples were stored at $-20{ }^{\circ} \mathrm{C}$ in a refrigerator.

\section{PCR amplification}

All DNA preparations were analyzed for the presence of E. bieneusi by nested PCR amplification of the ITS region of the rRNA gene with a set of specific primers. Primers and cycle parameters were designed by Mirjalal [28]. TaKaRa Taq DNA 
Table 1. Prevalence and genotype distribution of Enterocytozoon bieneusi isolates in pigs and wild boars worldwide.

\begin{tabular}{|c|c|c|c|c|}
\hline $\begin{array}{l}\text { Continents and } \\
\text { countries }\end{array}$ & Hosts & $\%$ (positive/total) & Genotypes (no.) & Ref. \\
\hline \multicolumn{5}{|l|}{ Americas } \\
\hline Brazil & Pigs & $59.3(54 / 91)$ & $\begin{array}{l}\text { EbpA (7), O (3), PigEb10 (1), H (1), PigEb2 } \\
\text { (16), PigEb4 (16), CS-1 (7), PigEb1 (5), } \\
\text { PigEb6 (2), PigEb3, PigEb5, PigEb7 - PigEb9, } \\
\text { and PigEb11 - PigEb17 (1 each) }\end{array}$ & [8] \\
\hline Peru & Pigs & $100.0(3 / 3)^{\mathrm{a}}$ & EbpC (3) & [7] \\
\hline USA & Pigs & $31.7(64 / 202)$ & $\begin{array}{l}\text { D, F, PigEBITS5, PigEBITS7, PigEBITS9 } \\
\text { and PigEBITS1 - PigEBITS4, PigEBITS6 } \\
\text { and PigEBITS8 (17) }\end{array}$ & {$[4]$} \\
\hline \multicolumn{5}{|c|}{$x^{-3}$} \\
\hline \multirow[t]{2}{*}{ China } & Pigs & $47.3(2787 / 5887)$ & $\begin{array}{l}\text { EbpC (1140), EbpA(440), CS-4 (58), } \\
\text { PigEBITS5 (53), O (51), D(47), Henan-IV } \\
\text { (43), H (40), LW1 (17), CS-1 (17), CHS5 (15), } \\
\text { CM8 (11), EbpD (7), CHN1 (4), KIN-1 (3), } \\
\text { Henan-III (2), I (2), Henan-I (1), CHG23 (1), } \\
\text { CM6 (1), SZZD1 (81), SLTC2 (59), CHG19 } \\
\text { (57), SYLA5 (56), EbpB (41), CHC5 (37), } \\
\text { SLTC3 (15), PigEB4 (15), CHN7 (14), G (13), } \\
\text { CM11 (10), CS-9 (9), SZZA2 (8), HN-1 (6), } \\
\text { CS-8 (5), CS-7 (4), XZP-II (4), CS-3 (3), FJF } \\
\text { (3), SZZC1 (3), WildBoar8 (3), CHN10, CS-6, } \\
\text { HLJ-I, CC-1, SLTC1, SYLA1, SHZA1, HN-2, } \\
\text { and XJP-II (2 each), CHN8, CHN9, CS-2, } \\
\text { CS-5, CS-10, CHN-RR2, CHG7, FJS, PigITS, } \\
\text { PigEBITS3, HLJ-II, HLJ-III, HLJ-IV, SYLA2, } \\
\text { SYLA2, SMXBB1, SMXC1, SZZB1, SZZA1, } \\
\text { SYLA3, SMXD1, SYLA4, SYLD1, CHG3, } \\
\text { SZZD2, SHZC1, SMXD2, SYLC1, HN-3, } \\
\text { HN-4, ZJ1, ZJ2, YN1-3, GD1, XZP-I, SCT01, } \\
\text { SCT02, and XJP-III (1 each) }\end{array}$ & $\begin{array}{l}{[18,19,22,24-26} \\
46,48,49,53,56 \\
57,62]\end{array}$ \\
\hline & Wild boars & $41.2(147 / 357)$ & $\begin{array}{l}\text { EbpC (85), EbpA (22), SH8 (6), D (1), LW1 (1), } \\
\text { PigEBITS5 (1), PigEBITS4 (11), CHC5 (10), } \\
\text { WildBoar8 (7), PigEBITS1, SC02, and } \\
\text { WildBoar11 (1 each) }\end{array}$ & {$[21]$} \\
\hline Japan & Pigs & $33.3(10 / 30)$ & $\begin{array}{l}\text { H (4), EbpC (2), D, EbpA, } \\
\text { and PigEBITS5 (1 each), Mixed/unknown (1) }\end{array}$ & {$[1]$} \\
\hline Korea & Pigs & $14.2(67 / 472)$ & $\begin{array}{l}\text { PigEBITS5 (4), CAF1 (1), H (1), PigEBITS3 (2), } \\
\text { and PigEBITS4 (2) }\end{array}$ & [12] \\
\hline Thailand & Pigs & $22.4(176 / 787)$ & $\begin{array}{l}\text { O (38), E (36), EbpC (20), H (8), F (8), LW1 (6), } \\
\text { D (5), EbpA (1), WildBoar5 (9), TMP1 }{ }^{\mathrm{b}}(3), \\
\text { TMP6 }^{\mathrm{b}}(2), \text { PigAYE1 }(2), \mathrm{TMP}^{\mathrm{b}}-\mathrm{TMP}^{\mathrm{b}}, \\
\text { TMP7 }^{\mathrm{b}}-\mathrm{TMP} 11^{\mathrm{b}}, \text { PigAYE} 2-3, \text { CS-10, } \\
\text { and SHZC1 }(1 \text { each) }\end{array}$ & {$[17,30,43,44]$} \\
\hline \multicolumn{5}{|l|}{ Europe } \\
\hline Austria & Wild boar & $13.6(6 / 44)$ & Henan-I (3), EbpC (2), D (1) & [29] \\
\hline \multirow[t]{2}{*}{ Czech Republic } & Pigs & $93.7(74 / 79)$ & F (70), D (2), Peru9 (2) & {$[35]$} \\
\hline & Wild boar & $8.7(20 / 231)$ & $\begin{array}{l}\text { EpbA (10), D (5), EbpC (1), WildBoar4 (2), G } \\
\text { (1), and WildBoar3 (1) }\end{array}$ & [29] \\
\hline Germany & Pigs & $23.3(21 / 90)$ & $\begin{array}{l}\text { F (7), H (2), O (4), E (1), Bfrmr2 (1), G (2), E1, } \\
\quad \text { F1, and Bfrmr5 (1 each) }\end{array}$ & {$[6,33,34]$} \\
\hline Poland & Wild boar & $7.6(10 / 129)$ & $\begin{array}{l}\text { EbpC (3), EbpA (2), WildBoar5 (2), WildBoar2, } \\
\text { WildBoar3, and WildBoar6 (1 each) }\end{array}$ & [29] \\
\hline Slovak Republic & Wild boar & $3.6(2 / 56)$ & D (1), WildBoar1 (1) & [29] \\
\hline Spain & Pigs & $20.6(7 / 34)$ & I (1) & [9] \\
\hline Switzerland & Pigs & $25.7(28 / 109)$ & EbpA (12), EbpC (7), EbpD (3), EbpB (6) & {$[3]$} \\
\hline
\end{tabular}

The genotypes found in humans previously are shown in bold.

${ }^{a}$ Genotyping study using confirmed E. bieneusi-positive isolates.

${ }^{\mathrm{b}}$ Invalid genotypes as the sequences submitted to GenBank contain an incomplete ITS region. 
Table 2. Prevalence and genotype distribution of E. bieneusi isolates in farm-raised pigs in Hainan Province.

\begin{tabular}{lccc}
\hline Category & Positive/examined (\%) & \multicolumn{1}{c}{ Genotype/s (no.) } & \% zoonotic genotypes \\
\hline Location & $16 / 30(53.3)$ & MJ14 (12), CS-4 (2), HNP-I (1), HNP-II (1) & 12.5 \\
$\quad$ Baisha & $20 / 58(34.5)$ & CS-4 (10), CHG19 (9), HNP-III (1) & 50.0 \\
Danzhou & $11 / 20(55.0)$ & CS-4 (10), EbpA (1) & 100.0 \\
Haikou & $41 / 80(51.3)$ & CS-4 (37), EbpA (3), HNP-IV (1) & 97.6 \\
Lingshui & & & 88.9 \\
Age & $36 / 61(54.5)$ & CS-4 (28), CHG19 (4), EbpA (4) & 59.6 \\
$\leq 60 \mathrm{~d}$ & $52 / 127(40.9)$ & CS-4 (31), MJ14 (12), CHG19 (5), HNP-I - HNP -IV (1 each) & 71.6 \\
$>60 \mathrm{~d}$ & $88 / 188(46.8)$ & CS-4 (59), MJ14 (12), CHG19 (9), EbpA (4), HNP-I - HNP-IV (1 each) & \\
Total & & &
\end{tabular}

Polymerase (TaKaRa Bio Inc., Tokyo, Japan) was used for all PCR amplifications at a final volume of $25 \mu \mathrm{L}$. A positive control with rat-derived genotype Peru 8 DNA and a negative control with no added DNA were amplified in all PCR tests. All secondary PCR products were subjected to electrophoresis on $1.5 \%$ agarose gels and visualized by staining with GelRed (Biotium Inc., Hayward, CA, USA).

\section{Nucleotide sequencing and analyzing}

All secondary PCR products positive for E. bieneusi were sequenced by Sangon Biotech Co., Ltd. (Shanghai, China). Sequence accuracy was confirmed by two-directional sequencing and by sequencing additional PCR products, as required. The genotypes of E. bieneusi were identified through the comparison of nucleotide sequences obtained with each other, and from published GenBank sequences using the Basic Local Alignment Search Tool (BLAST) (http://blast.ncbi.nlm.nih. gov/Blast.cgi) and ClustalX 1.83 (http://www.clustal.org/). The obtained genotypes of E. bieneusi were given a published name when identical to the genotypes in GenBank [40]. In parallel, the genotypes that produced ITS sequences with any single nucleotide substitutions, deletions, or insertions were confirmed by DNA sequencing of at least two PCR products and considered as novel genotypes. All were given a genotype name through the addition of roman numerals behind the abbreviation HNP (Hainan Pig), according to their order of appearance. All genotypes were named based on a 243 bp sequence of the ITS gene region of E. bieneusi, according to the established nomenclature system [40].

\section{Phylogenetic analysis}

To confirm the genogroup designation and to assess the genetic relationships of obtained novel ITS genotypes of E. bieneusi with known genotypes, a phylogenetic analysis was performed through the construction of a neighbor-joining tree using the program Mega X (http://www.megasoftware. net/) based on the evolutionary distances calculated by the Kimura-2-parameter model. The reliability of these trees was assessed using bootstrap analysis with 1000 replicates.

\section{Statistical analysis}

Differences in the infection rates among different locations and ages were assessed using a Chi-square test with SPSS
Version 22.0 software (IBM Corp., Armonk, NY, USA). $P$-values $<0.05$ were considered significant.

\section{Nucleotide sequence accession numbers}

Representative nucleotide sequences obtained in the study were deposited in the GenBank database under accession numbers MN630620-MN630623.

\section{Results}

\section{Occurrence of $E$. bieneusi in pigs}

E. bieneusi was detected in $46.8 \%$ (88/188) of the pig samples. The pathogen was detected in all four cities in Hainan Province, with peak infection rates of $55.0 \%(11 / 20)$ in Haikou, followed by Baisha (53.3\%; 16/30), Lingshui $(51.3 \%$; 41/80), and Danzhou $(34.5 \%, 20 / 58)$ (Table 2). There were no significant differences in the prevalence among different locations $\left(\chi^{2}=2.614, p>0.05\right)$. Regarding the two age groups, the infection rate in the younger group (aged $\leq 60$ days) was $54.5 \%(36 / 61)$, which was significantly higher than that in the older group (aged $>60$ days) at $40.9 \% \quad(52 / 127)$ $\left(\chi^{2}=5.405, p<0.05\right)$ (Table 2).

\section{Genetic characterizations and genotypic distribution of $E$. bieneusi in pigs}

Through sequence analysis of 88 E. bieneusi isolates, eight ITS genotypes were identified with a total of 16 polymorphic sites observed among them (Table 3). Four known genotypes (CS-4, MJ14, CHG19, and EbpA) and four novel genotypes termed HNP-I - HNP-IV were identified (Table 2). Among the genotypes, CS-4 was the most prevalent and identified in $59(59 / 88,67.0 \%)$ positive specimens, followed by MJ14, CHG19, and EbpA found in 12 (12/88, 13.6\%), 9 (8/88, $10.2 \%)$, and $4(4 / 88,4.5 \%)$ specimens, respectively. Genotypes HNP-I - HNP-IV were identified in one specimen each (Table 2).

Genotypes HNP-I (MN630621) and HNP-II (MN630622) had the largest similarities with genotype MJ14 (MK348513), while genotype HNP-I (MN630621) had a single nucleotide (C) insertion at position 2. Genotype HNP-II (MN630622) had one base variation at position $148(\mathrm{G} \rightarrow \mathrm{A})$. In contrast, genotypes HNP-III (MN630620) and HNP-IV (MN630623) had one base difference compared to genotypes CHG19 
Table 3. Variation of the ITS gene sequences of E. bieneusi isolates in farm-raised pigs in Hainan Province.

\begin{tabular}{|c|c|c|c|c|c|c|c|c|c|c|c|c|c|c|c|c|c|}
\hline \multirow[t]{2}{*}{ Genotypes } & \multirow{2}{*}{$\begin{array}{c}\text { GenBank } \\
\text { accession no. }\end{array}$} & \multicolumn{16}{|c|}{ Nucleotide at position } \\
\hline & & 2 & 11 & 14 & 31 & 32 & 82 & 96 & 132 & 137 & 138 & 142 & 144 & 148 & 159 & 197 & 224 \\
\hline \multicolumn{18}{|l|}{ Known } \\
\hline $\mathrm{CS}-4$ & MK778898 & - & $\mathrm{G}$ & $\mathrm{G}$ & $\mathrm{T}$ & $\mathrm{G}$ & $\mathrm{C}$ & $\mathrm{G}$ & $\mathrm{G}$ & $\mathrm{G}$ & $\mathrm{C}$ & $\mathrm{C}$ & A & $\mathrm{G}$ & $\mathrm{T}$ & A & $\mathrm{C}$ \\
\hline CHG19 & MH817463 & - & $\mathrm{G}$ & $\mathrm{G}$ & $\mathrm{T}$ & A & $\mathrm{C}$ & $\mathrm{T}$ & $\mathrm{G}$ & $\mathrm{G}$ & $\mathrm{C}$ & $\mathrm{C}$ & $\mathrm{A}$ & $\mathrm{G}$ & $\mathrm{T}$ & A & $\mathrm{C}$ \\
\hline EbpA & AF076040 & - & $\mathrm{G}$ & $\mathrm{G}$ & $\mathrm{T}$ & $\mathrm{A}$ & $\mathrm{T}$ & $\mathrm{T}$ & $\mathrm{G}$ & A & $\mathrm{C}$ & $\mathrm{T}$ & $\mathrm{G}$ & $\mathrm{G}$ & $\mathrm{T}$ & $\mathrm{G}$ & $\mathrm{C}$ \\
\hline MJ14 & MK348513 & - & A & $\mathrm{G}$ & $\mathrm{C}$ & A & $\mathrm{T}$ & $\mathrm{G}$ & $\mathrm{C}$ & G & $\mathrm{T}$ & $\mathrm{T}$ & A & G & $\mathrm{G}$ & A & $\mathrm{C}$ \\
\hline \multicolumn{18}{|l|}{ Novel } \\
\hline HNP-I & MN630621 & $\mathrm{C}$ & A & $\mathrm{G}$ & $\mathrm{C}$ & A & $\mathrm{T}$ & $\mathrm{G}$ & $\mathrm{C}$ & G & $\mathrm{T}$ & $\mathrm{T}$ & A & G & G & A & $\mathrm{C}$ \\
\hline HNP-II & MN630622 & - & A & $\mathrm{G}$ & $\mathrm{C}$ & A & $\mathrm{T}$ & $\mathrm{G}$ & $\mathrm{C}$ & $\mathrm{G}$ & $\mathrm{T}$ & $\mathrm{T}$ & A & $\mathrm{A}$ & $\mathrm{G}$ & A & $\mathrm{C}$ \\
\hline HNP-III & MN630620 & - & $\mathrm{G}$ & A & $\mathrm{T}$ & A & $\mathrm{C}$ & $\mathrm{T}$ & $\mathrm{G}$ & G & $\mathrm{C}$ & $\mathrm{C}$ & A & $\mathrm{G}$ & $\mathrm{T}$ & A & $\mathrm{C}$ \\
\hline HNP-IV & MN630623 & - & $\mathrm{G}$ & $\mathrm{G}$ & $\mathrm{T}$ & $\mathrm{G}$ & $\mathrm{C}$ & $\mathrm{G}$ & $\mathrm{G}$ & $\mathrm{G}$ & $\mathrm{C}$ & $\mathrm{C}$ & $\mathrm{A}$ & $\mathrm{G}$ & $\mathrm{T}$ & $\mathrm{A}$ & $\mathrm{T}$ \\
\hline
\end{tabular}

$(\mathrm{MH} 817463)$ and CS-4 (MK778898) at positions $13(\mathrm{G} \rightarrow \mathrm{A})$ and $223(\mathrm{C} \rightarrow \mathrm{T})$, respectively.

Genotype CS-4 was identified in all four of the locations with EbpA in Haikou and Lingshui, MJ14, HNP-I, and HNP-II in Baisha, CHG19 and HNP-III in Danzhou, and HNP-IV in Lingshui, respectively. Considering the two age groups, genotypes CS-4 and CHG19 were found in both age groups, while genotype EbpA was exclusively found in the younger group. Genotypes HNP-I - HNP-IV and MJ14 were exclusive to the older groups (Table 2).

\section{Phylogenetic relationship of $E$. bieneusi genotypes}

Based on the phylogenetic analysis of the neighbor-joining tree of the ITS gene sequences of E. bieneusi, genotypes CS-4, CHG19, EbpA, HNP-III, and HNP-IV were located in zoonotic Group 1, while genotypes MJ14, HNP-I, and HNP-II were clustered into Group 10 (Fig. 2).

\section{Discussion}

In this study, the overall infection rate of E. bieneusi in pigs was $46.8 \%$ (88/188), which was similar to that reported in other provinces of China, such as Heilongjiang $(45.3 \%$; 39/86) [22], Henan (45.5\%; 408/897) [48], Tibet (43.2\%; 309/715) [18], and Xinjiang $(48.6 \%$; 389/801) [19]. A total of 30 studies on E. bieneusi in pigs or wild boars from 14 countries have been reported with average infection rates of $42.3 \%$ (3291/7784) in pigs and $22.6 \%(185 / 817)$ in wild boars. Among them, 14 of the studies are from China with an average infection rate of $47.0 \%$ (2934/6244), ranging from 10.2\% (5/49) in Heilongjiang to $100 \%(2 / 2)$ in Inner Mongolia. The prevalence of E. bieneusi in pigs from developed countries has been reported at rates ranging from $3.6 \%(2 / 56)$ to $93.7 \%(74 / 79)$, which is lower than rates reported in developing countries (10.2-100.0\%).

As for other opportunistic pathogens, the prevalence of E. bieneusi is closely associated with host age. In the present study, younger pigs $(54.5 \%$; 36/61) had significantly higher infection rates of $E$. bieneusi than older pigs $(40.9 \%$; 52/127) $\left(\chi^{2}=5.405, P<0.05\right)$, in agreement with previous studies. In studies from Thailand, the prevalence of E. bieneusi in pigs aged 2-3.9 months was significantly higher than other age

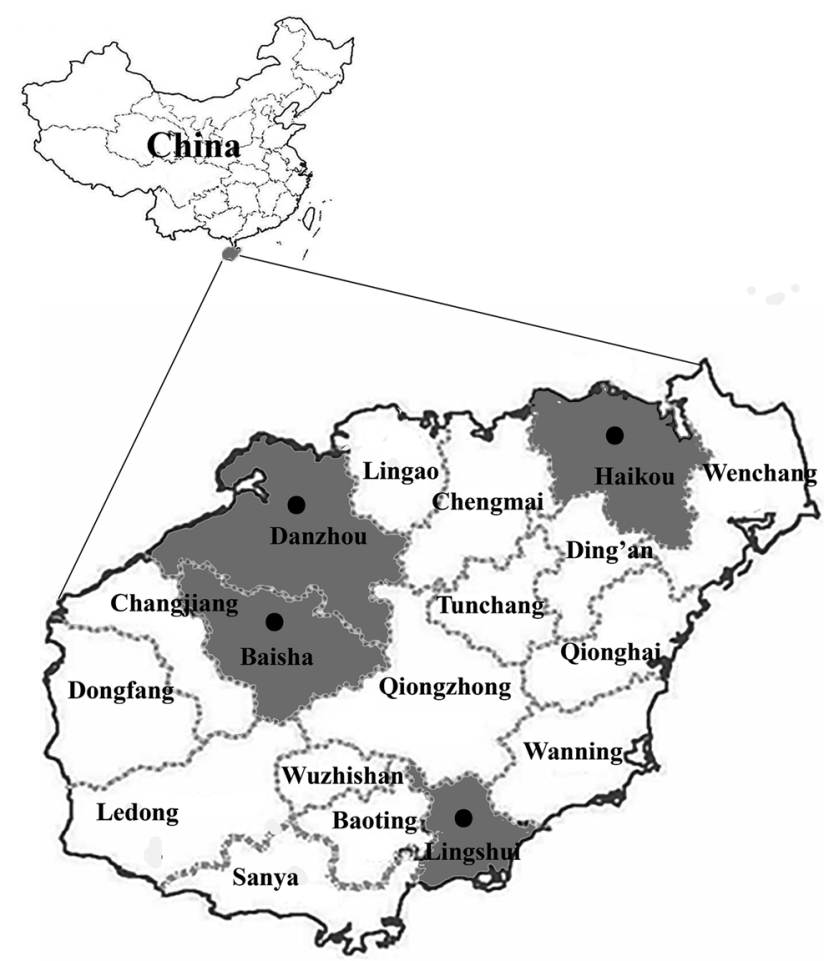

Figure 1. Specific locations where samples were collected in this study. Dots indicate sampling points.

groups [17]. Likewise, Li et al. reported that among the three age groups, the nursery group had the highest prevalence rates of E. bieneusi compared to those in the pre-weaned and growing groups [22]. Meanwhile, Zhao et al. showed that postweaned piglets aged 2-3 months presented high infection rates (89.5\%) [57]. The prevalence of E. bieneusi in young pigs was higher than that of adults, most likely due to the underdeveloped immune systems of the young animals.

In the present study, eight genotypes of E. bieneusi were identified with four previously reported genotypes CS-4, MJ14, CHG19, and EbpA, and four novel genotypes HNP-I - HNP-IV. Among them, genotype CS-4 was dominant with the highest occurrence $(67.0 \% ; 59 / 88)$ and the widest distribution (detected in all four investigated areas) in pigs from Hainan. This genotype has also been reported in other animals, 


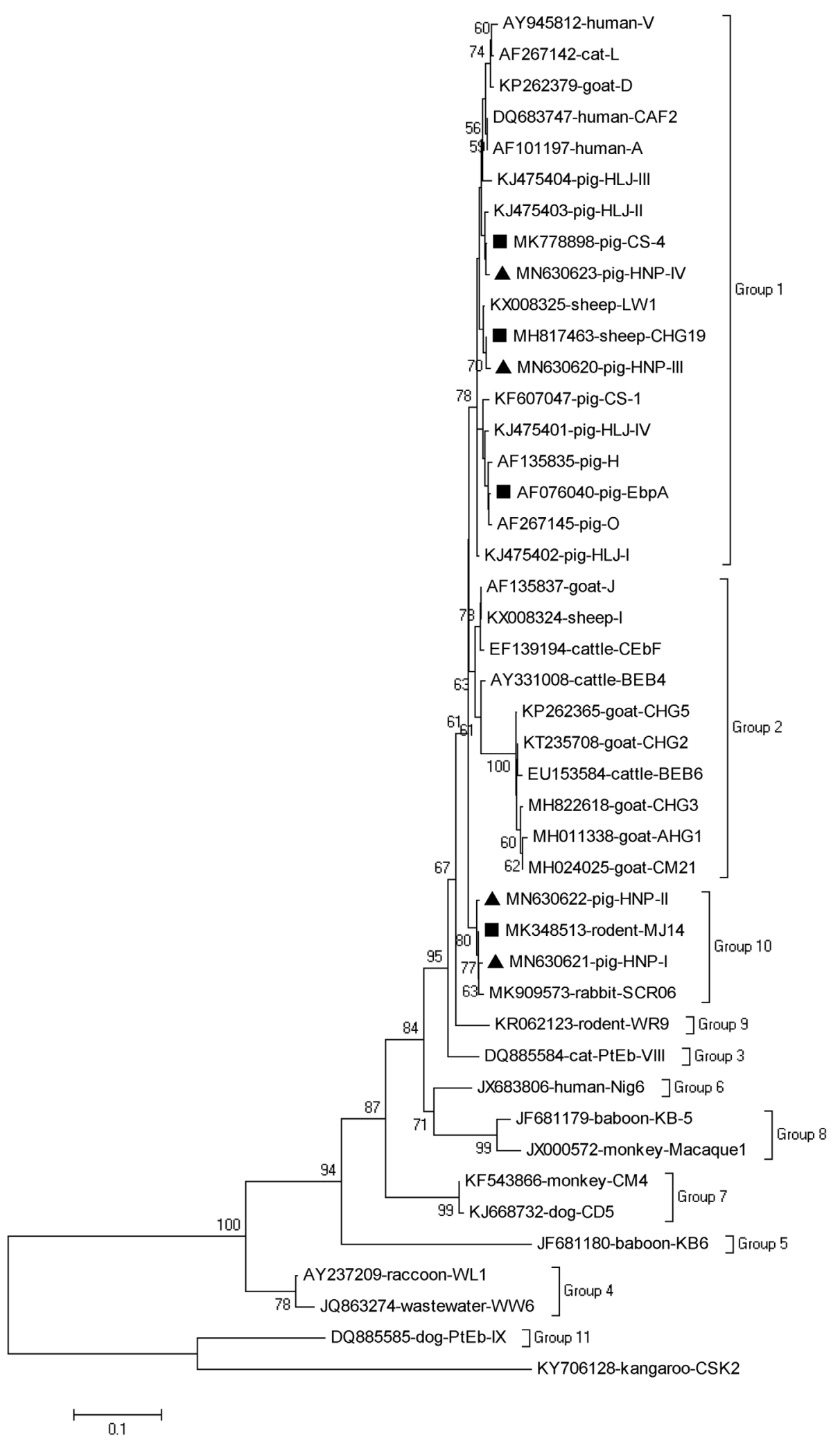

Figure 2. Phylogenetic tree based on the neighbor-joining analysis of ITS sequences. Phylogenetic relationships of E. bieneusi genotypes identified in pigs here and other known genotypes deposited in GenBank were inferred by a neighbor-joining analysis of ITS sequences based on genetic distance by the Kimura-2-parameter model. The numbers on the branches are percent bootstrapping values from 1000 replicates. Each sequence is identified by its accession number, host origin, and genotype designation. The E. bieneusi genotype CSK2 (KY706128) from white kangaroo was used as the outgroup. The squares and triangles filled in black indicate novel and known genotypes identified in this study, respectively. 
including non-human primates [14], sheep [13], and horses [32]. It was shown that genotype CS-4 has the ability to infect humans, particularly children [51], and has been found in river water [11]. Genotype EbpA was identified in four pigs in this study. A large variety of animals have been reported to be infected with genotype EbpA. In addition to pigs, EbpA has been detected in non-human primates $[37,39]$, cattle $[5,6$, $11,58]$, sheep [42], goats [42, 59], deer [54], horses [32, 45], house mice [36], and birds [15, 16], with a wide host range. This genotype has also been found in humans from the Czech Republic [38], Nigeria [2], and China [47], highlighting its zoonotic nature. Recently, Li et al. reported that genotype EbpA is present on the surfaces of vegetables and fruits, highlighting a possible risk of foodborne-related disease outbreaks [23].

The other two known genotypes (MJ14 and CHG19) identified in this study have only been found in animals to date. To our knowledge, genotype MJ14 (MK348513) was originally identified and designated in binturong (Arctictis binturong) from Yunnan Province (unpublished). It was also termed HNR-VI (MN267057) and found in Asiatic brush-tailed porcupines from Hainan Province (unpublished). The identification of genotype MJ14 (HNR-VI) in pigs and rodents in the same province not only suggests that the genotype has an extensive host range, but that potential cross-species transmission between pigs and rodents can occur. Genotype CHG19 has been identified in pigs from Henan [48] and Shaanxi [49], in captive Eurasian wild boars from Sichuan [21], in goats from Yunnan [42], in horses from Xinjiang [32] and on the surfaces of vegetables and fruits from Henan [23]. This suggests that CHG19 has the ability to infect a large variety of animals. Despite the lack of detection of CHG19 in humans, it should continue to be monitored.

Four novel genotypes were identified in this study. The number of ITS genotypes of E. bieneusi is increasing dramatically with a larger number of isolates of $E$. bieneusi sequenced. It is conceivable that if sufficient isolates are sequenced, all nucleotides in the ITS could be polymorphic [4]. Meanwhile, although the ITS region is $243 \mathrm{bp}$ in length for the majority of E. bieneusi genotypes, length variations of the ITS region of rDNA of $E$. bieneusi have been identified in five genotypes, with one or two nucleotide deletions (241 bp or $242 \mathrm{bp}$ ) [52, 53], and three genotypes with a single nucleotide insertion ( $244 \mathrm{bp}$ ) in the ITS region [52, 60]. It was observed that there were 244 bp in the ITS region of genotype HNP-I. Other genetic markers are required to substantiate these observations.

Currently, 95.5\% (128/134) of the genotypes identified in pigs or wild boars were clustered into Group 1, while the other six genotypes (I, CHN1, CHN9, CHN10, SZZD2, and CHG3) clustered into Group 2 (Table 1). Genotypes CS-4, CHG19, and EbpA, and two novel genotypes (HNP-III and HNP-IV) were located in zoonotic Group 1, while genotype MJ14 (MK348513) and the other two novel genotypes (HNP-I and HNP-II) were clustered into Group 10.

\section{Conclusions}

This is the first study to report the identification of E. bieneusi in pigs in Hainan Province, with a high prevalence and wide occurrence demonstrated (detected in all four investigated areas). The findings that the two known humanpathogenic genotypes (CS-4 and EbpA) are in high proportions, and that genotype CHG19 as well as two novel genotypes (HNP-III and HNP-IV) of E. bieneusi belong to zoonotic Group 1, indicate the possibility of transmission between pigs and humans. This study represents the first identification of genotypes in Group 10 (MJ14, HNP-I, and HNP-II) in pigs, indicating the unique epidemic features of E. bieneusi in pigs in Hainan Province.

\section{Competing interests}

The authors declare that they have no competing interests.

Acknowledgements. This work was supported by the Innovation Research Team Project of the Hainan Natural Science Foundation (No. 2018CXTD340), the National Natural Science Foundation of China (Nos. 81672072 and 81760378) and the Graduate Student Innovation Foundation of colleges and universities of Hainan Province, 2019 (No. Hys2019-287). The funders had no role in study design, data collection and analysis, the decision to publish, or preparation of the manuscript.

\section{References}

1. Abe N, Kimata I. 2010. Molecular survey of Enterocytozoon bieneusi in a Japanese porcine population. Vector-Borne and Zoonotic Disease, 10(4), 425-427.

2. Akinbo FO, Okaka CE, Omoregie R, Dearen T, Leon ET, Xiao L. 2012. Molecular epidemiologic characterization of Enterocytozoon bieneusi in HIV-infected persons in Benin City, Nigeria. American Journal of Tropical Medicine and Hygiene, 86(3), 441-445.

3. Breitenmoser AC, Mathis A, Bürgi E, Weber R, Deplazes P. 1999. High prevalence of Enterocytozoon bieneusi in swine with four genotypes that differ from those identified in humans. Parasitology, 118, 447-453.

4. Buckholt MA, Lee JHA, Tzipori S. 2002. Prevalence of Enterocytozoon bieneusi in swine: an 18-month survey at a slaughterhouse in Massachusetts. Applied \& Environmental Microbiology, 68(5), 2595-2599.

5. da Silva Fiuza VR, Lopes CW, de Oliveira FC, Fayer R, Santin M. 2016. New findings of Enterocytozoon bieneusi in beef and dairy cattle in Brazil. Veterinary Parasitology, 216, $46-51$.

6. Dengjel B, Zahler M, Hermanns W, Heinritzi K, Spillmann T, Thomschke A, Löscher T, Gothe R, Rinder H. 2001. Zoonotic potential of Enterocytozoon bieneusi. Journal of Clinical Microbiology, 39(12), 4495-4499.

7. Feng Y, Li N, Dearen T, Lobo ML, Matos O, Cama V, Xiao L. 2011. Development of a multilocus sequence typing tool for high-resolution genotyping of Enterocytozoon bieneusi. Applied \& Environmental Microbiology, 77(14), 4822-4828.

8. Fiuza VR, Oliveira FC, Fayer R, Santín M. 2015. First report of Enterocytozoon bieneusi in pigs in Brazil. Parasitology International, 64(4), 18-23.

9. Galván-Díaz AL, Magnet A, Fenoy S, Henriques-Gil N, Haro M, Gordo FP, Millán J, Miró G, del Águila C, Izquierdo F. 2014. Microsporidia detection and genotyping study of human pathogenic Enterocytozoon bieneusi in animals from Spain. PLoS One, 9(3), e92289. 
10. Gong B, Yang Y, Liu X, Cao J, Xu M, Xu N, Yang F, Wu F, Li B, Liu A, Shen Y. 2019. First survey of Enterocytozoon bieneusi and dominant genotype Peru6 among ethnic minority groups in southwestern China's Yunnan Province and assessment of risk factors. PLoS Neglected Tropical Diseases, 13(5), e0007356.

11. Hu Y, Feng Y, Huang C, Xiao L. 2014. Occurrence, source, and human infection potential of Cryptosporidium and Enterocytozoon bieneusi in drinking source water in Shanghai, China, during a pig carcass disposal incident. Environmental Science \& Technology, 48(24), 14219-14227.

12. Jeong DK, Won GY, Park BK, Hur J, You JY, Kang SJ, Oh IG, Lee YS, Stein BD, Lee JH. 2007. Occurrence and genotypic characteristics of Enterocytozoon bieneusi in pigs with diarrhea. Parasitology Research, 102(1), 123-128.

13. Jiang Y, Tao W, Wan Q, Li Q, Yang Y, Lin Y, Zhang S, Li W. 2015. Zoonotic and potentially host-adapted Enterocytozoon bieneusi genotypes in sheep and cattle in Northeast China and an increasing concern about the zoonotic importance of previously considered ruminant-adapted genotypes. Applied \& Environmental Microbiology, 81(10), 3326-3335.

14. Karim MR, Wang R, Dong H, Zhang L, Li J, Zhang S, Rume FI, Qi M, Jian F, Sun M, Yang G, Zou F, Ning C, Xiao L. 2014. Genetic polymorphism and zoonotic potential of Enterocytozoon bieneusi from nonhuman primates in China. Applied \& Environmental Microbiology, 80(6), 1893-1898.

15. Kasicková D, Sak B, Kvác M, Ditrich O. 2009. Sources of potentially infectious human microsporidia: molecular characterisation of microsporidia isolates from exotic birds in the Czech Republic, prevalence study and importance of birds in epidemiology of the human microsporidial infections. Veterinary Parasitology, 165(1-2), 125-130.

16. Lallo MA, Calábria P, Milanelo L. 2012. Encephalitozoon and Enterocytozoon (Microsporidia) spores in stool from pigeons and exotic birds: microsporidia spores in birds. Veterinary Parasitology, 190(3-4), 418-422.

17. Leelayoova S, Piyaraj P, Subrungruang I, Pagornrat W, Naaglor T, Phumklan S, Taamasri P, Suwanasri J, Mungthin M. 2009. Genotypic characterization of Enterocytozoon bieneusi in specimens from pigs and humans in a pig farm community in Central Thailand. Journal of Clinical Microbiology, 47(5), 15721574.

18. Li D, Zheng S, Zhou C, Karim MR, Wang L, Wang H, Yu F, Li J, Wang W, Wang Y, Zhang S, Jian F, Wang R, Ning C, Zhang L. 2019. Multilocus typing of Enterocytozoon bieneusi in pig reveals the high prevalence, zoonotic potential, host adaptation and geographical segregation in China. Journal of Eukaryotic Microbiology, 66(5), 707-718.

19. Li D, Zhang Y, Jiang Y, Xing J, Tao D, Zhao A, Cui Z, Jing B, Qi M, Zhang L. 2019. Genotyping and zoonotic potential of Enterocytozoon bieneusi in pigs in Xinjiang, China. Front Microbiology, 10, 2401.

20. Liu H, Jiang Z, Yuan Z, Yin J, Wang Z, Yu B, Zhou D, Shen Y, Cao J. 2017. Infection by and genotype characteristics of Enterocytozoon bieneusi in HIV/AIDS patients from Guangxi Zhuang autonomous region, China. BMC Infectious Diseases, 17(1), 684 .

21. Li W, Deng L, Wu K, Huang X, Song Y, Su H, Hu Y, Fu H, Zhong Z, Peng G. 2017. Presence of zoonotic Cryptosporidium scrofarum, Giardia duodenalis assemblage A and Enterocytozoon bieneusi genotypes in captive Eurasian wild boars (Sus scrofa) in China: potential for zoonotic transmission. Parasites \& Vectors, 10(1), 10.

22. Li W, Diao R, Yang J, Xiao L, Lu Y, Li Y, Song M. 2014. High diversity of human-pathogenic Enterocytozoon bieneusi genotypes in swine in northeast China. Parasitology Research, $113,1147-1153$.

23. Li W, Feng Y, Santin M. 2019. Host specificity of Enterocytozoon bieneusi and public health implications. Trends in Parasitology, 35(6), 436-451.

24. Li W, Li Y, Li W, Yang J, Song M, Diao R, Jia H, Lu Y, Zheng J, Zhang X, Xiao L. 2014. Genotypes of Enterocytozoon bieneusi in livestock in China: high prevalence and zoonotic potential. PLoS One, 9(5), e97623.

25. Li W, Tao W, Jiang Y, Diao R, Yang J, Xiao L. 2014. Genotypic distribution and phylogenetic characterization of Enterocytozoon bieneusi in diarrheic chickens and pigs in multiple cities, China: potential zoonotic transmission. PLoS One, 9(9), e108279.

26. Luo R, Xiang L, Liu H, Zhong Z, Liu L, Deng L, Liu L, Huang X, Zhou Z, Fu H, Luo Y, Peng G. 2019. First report and multilocus genotyping of Enterocytozoon bieneusi from Tibetan pigs in southwestern China. Parasite, 26, 24.

27. Matos O, Lobo ML, Xiao L. 2012. Epidemiology of Enterocytozoon bieneusi infection in humans. Journal of Parasitology Research, 2012, 981424.

28. Mirjalali H, Mirhendi H, Meamar AR, Mohebali M, Askari Z, Mirsamadi ES, Rezaeian M. 2015. Genotyping and molecular analysis of Enterocytozoon bieneusi isolated from immunocompromised patients in Iran. Infection, Genetics and Evolution, 36, 244-249.

29. Němejc K, Sak B, Květoňová D, Hanzal V, Janiszewski P, Forejtek P, Rajský D, Kotková M, Ravaszová P, McEvoy J, Kváč M. 2014. Prevalence and diversity of Encephalitozoon spp. and Enterocytozoon bieneusi in wild boars (Sus scrofa) in Central Europe. Parasitology Research, 113(2), 761-767.

30. Prasertbun R, Mori H, Pintong AR, Sanyanusin S, Popruk S, Komalamisra C, Changbunjong T, Buddhirongawatr R, Sukthana Y, Mahittikorn A. 2017. Zoonotic potential of Enterocytozoon genotypes in humans and pigs in Thailand. Veterinary Parasitology, 233, 73-79.

31. Prasertbun R, Mori H, Sukthana Y, Popruk S, Kusolsuk T, Hagiwara K, Mahittikorn A. 2019. Enterocytozoon bieneusi and Cryptosporidium: a cross-sectional study conducted throughout Thailand. BMC Infectious Diseases, 19(1), 808.

32. Qi M, Wang R, Wang H, Jian F, Li J, Zhao J, Dong H, Zhu H, Ning C, Zhang L. 2016. Enterocytozoon bieneusi genotypes in grazing horses in China and their zoonotic transmission potential. Journal of Eukaryotic Microbiology, 63(5), 591-597.

33. Reetz J, Nöckler K, Reckinger S, Vargas MM, Weiske W, Broglia A. 2009. Identification of Encephalitozoon cuniculi genotype III and two novel genotypes of Enterocytozoon bieneusi in swine. Parasitology International, 58, 285-292.

34. Rinder H, Thomschke A, Dengjel B, Gothe R, Löscher T, Zahler M. 2000. Close genotypic relationship between Enterocytozoon bieneusi from humans and pigs and first detection in cattle. Journal of Parasitology, 86, 185-188.

35. Sak B, Kvác M, Hanzlíková D, Cama V. 2008. First report of Enterocytozoon bieneusi infection on a pig farm in the Czech Republic. Veterinary Parasitology, 153, 220-224.

36. Sak B, Kváč M, Květoňová D, Albrecht T, Piálek J. 2011. The first report on natural Enterocytozoon bieneusi and Encephalitozoon spp. infections in wild East-European House Mice (Mus musculus musculus) and West-European House Mice (M. m. domesticus) in a hybrid zone across the Czech RepublicGermany border. Veterinary Parasitology, 178(3-4), 246-250.

37. Sak B, Kvác M, Petrzelková K, Kvetonová D, Pomajbíková K, Mulama M, Kiyang J, Modrý D. 2011. Diversity of microsporidia (Fungi: Microsporidia) among captive great apes in 
European zoos and African sanctuaries: evidence for zoonotic transmission? Folia Parasitologica (Praha), 58(2), 81-86.

38. Sak B, Brady D, Pelikánová $M$, Květoňová D, Rost $M$, Kostka M, Tolarová V, Hůzová Z, Kváč M. 2011. Unapparent microsporidial infection among immunocompetent humans in the Czech Republic. Journal of Clinical Microbiology, 49(3), 1064-1070.

39. Sak B, Petrželková KJ, Květoňová D, Mynářová A, Pomajbíková K, Modrý D, Cranfield MR, Mudakikwa A, Kváč M. 2014. Diversity of microsporidia, Cryptosporidium and Giardia in mountain gorillas (Gorilla beringei beringei) in Volcanoes National Park, Rwanda. PLoS One, 9(11), e109751.

40. Santín M, Fayer R. 2009. Enterocytozoon bieneusi genotype nomenclature based on the internal transcribed spacer sequence: a consensus. Journal of Eukaryotic Microbiology, 56(1), 34-38.

41. Santín M, Fayer R. 2011. Microsporidiosis: Enterocytozoon bieneusi in domesticated and wild animals. Research in Veterinary Science, 90(3), 363-371.

42. Shi K, Li M, Wang X, Li J, Karim MR, Wang R, Zhang L, Jian F, Ning C. 2016. Molecular survey of Enterocytozoon bieneusi in sheep and goats in China. Parasites \& Vectors, 9, 23.

43. Thathaisong U, Siripattanapipong S, Leelayoova S, Mungthin M. 2019. Prevalence and molecular characterization of Enterocytozoon bieneusi among pigs in Chonburi Province, Eastern Thailand. American Journal Tropical Medicine and Hygiene, 101, 1392-1396.

44. Udonsom R, Prasertbun R, Mahittikorn A, Chiabchalard R, Sutthikornchai C, Palasuwan A, Popruk S. 2019. Identification of Enterocytozoon bieneusi in goats and cattle in Thailand. BMC Veterinary Research, 15(1), 308.

45. Wagnerová $\mathrm{P}$, Sak $\mathrm{B}$, Květoňová $\mathrm{D}$, Buňatová $\mathrm{Z}$, Civišová $\mathrm{H}$, Maršálek M, Kváč M. 2012. Enterocytozoon bieneusi and Encephalitozoon cuniculi in horses kept under different management systems in the Czech Republic. Veterinary Parasitology, 190(3-4), 573-577.

46. Wan Q, Lin Y, Mao Y, Yang Y, Li Q, Zhang S, Jiang Y, Tao W, Li W. 2016. High prevalence and widespread distribution of zoonotic Enterocytozoon bieneusi genotypes in swine in Northeast China: implications for public health. Journal of Eukaryotic Microbiology, 63(2), 162-170.

47. Wang L, Xiao L, Duan L, Ye J, Guo Y, Guo M, Liu L, Feng Y. 2013. Concurrent infections of Giardia duodenalis, Enterocytozoon bieneusi, and Clostridium difficile in children during a cryptosporidiosis outbreak in a pediatric hospital in China. PLoS Neglected Tropical Diseases, 7(9), e2437.

48. Wang H, Zhang Y, Wu Y, Li J, Qi M, Li T, Wang J, Wang R, Zhang S, Jian F, Ning C, Zhang L. 2018. Occurrence, molecular characterization, and assessment of zoonotic risk of Cryptosporidium spp., Giardia duodenalis, and Enterocytozoon bieneusi in pigs in Henan, Central China. Journal of Eukaryotic Microbiology, 65(6), 893-901.

49. Wang SS, Li JQ, Li YH, Wang XW, Fan XC, Liu X, Li ZJ, Song JK, Zhang LX, Zhao GH. 2018. Novel genotypes and multilocus genotypes of Enterocytozoon bieneusi in pigs in northwestern China: a public health concern. Infection, Genetics and Evolution, 63, 89-94.
50. Wang SS, Wang RJ, Fan XC, Liu TL, Zhang LX, Zhao GH. 2018. Prevalence and genotypes of Enterocytozoon bieneusi in China. Acta Tropica, 183, 142-152.

51. Yang J, Song M, Wan Q, Li Y, Lu Y, Jiang Y, Tao W, Li W. 2014. Enterocytozoon bieneusi genotypes in children in Northeast China and assessment of risk of zoonotic transmission. Journal of Clinical Microbiology, 52(12), 4363-4367.

52. Yang Z, Zhao W, Shen Y, Zhang W, Shi Y, Ren G, Yang D, Ling H, Yang F, Liu A, Cao J. 2016. Subtyping of Cryptosporidium cuniculus and genotyping of Enterocytozoon bieneusi in rabbits in two farms in Heilongjiang Province, China. Parasite, 23, 52.

53. Zhang X, Wang Z, Su Y, Liang X, Sun X, Peng S, Lu H, Jiang N, Yin J, Xiang M, Chen Q. 2011. Identification and genotyping of Enterocytozoon bieneusi in China. Journal of Clinical Microbiology, 49(5), 2006-2008.

54. Zhang Z, Huang J, Karim MR, Zhao J, Dong H, Ai W, Li F, Zhang L, Wang R. 2015. Zoonotic Enterocytozoon bieneusi genotypes in Pere David's deer (Elaphurus davidianus) in Henan, China. Experimental Parasitology, 155, 46-48.

55. Zhang Y, Koehler AV, Wang T, Robertson GJ, Bradbury RS, Gasser RB. 2018. Enterocytozoon bieneusi genotypes in people with gastrointestinal disorders in Queensland and Western Australia. Infection, Genetics and Evolution, 65, 293-299.

56. Zhang N, Wu R, Ji T, Cui LL, Cao HX, Li D, Li J, Zhang L, Huang C, Zhou DH. 2019. Molecular detection, multilocus genotyping, and population genetics of Enterocytozoon bieneusi in pigs in Southeastern China. Journal of Eukaryotic Microbiology, 67, 1-8.

57. Zhao W, Zhang W, Yang F, Cao J, Liu H, Yang D, Shen Y, Liu A. 2014. High prevalence of Enterocytozoon bieneusi in asymptomatic pigs and assessment of zoonotic risk at the genotype level. Applied \& Environmental Microbiology, 80(12), 3699-3707.

58. Zhao W, Zhang W, Yang F, Zhang L, Wang R, Cao J, Shen Y, Liu A. 2015. Enterocytozoon bieneusi in dairy cattle in the Northeast of China: genetic diversity of ITS gene and evaluation of zoonotic transmission potential. Journal of Eukaryotic Microbiology, 62(4), 553-560.

59. Zhao W, Zhang W, Yang D, Zhang L, Wang R, Liu A. 2015. Prevalence of Enterocytozoon bieneusi and genetic diversity of ITS genotypes in sheep and goats in China. Infection, Genetics and Evolution, 32, 265-270.

60. Zhao W, Zhou H, Jin H, Sun L, Li P, Liu M, Qiu M, Xu L, Li F, Ma T, Wang S, Yin F, Li L, Cui X, Chan JF, Lu G. 2019. Genotyping of Enterocytozoon bieneusi among captive longtailed macaques (Macaca fascicularis) in Hainan Province: high genetic diversity and zoonotic potential. Acta Tropica, 201, 105211.

61. Zhou H, Zheng X, Ma T, Qi M, Cao Z, Chao Z, Wei L, Liu Q, Sun R, Wang F, Zhang Y, Lu G, Zhao W. 2019. Genotype identification and phylogenetic analysis of Enterocytozoon bieneusi in farmed black goats (Capra hircus) from China's Hainan Province. Parasite, 26, 62.

62. Zou Y, Hou JL, Li FC, Zou FC, Lin RQ, Ma JG, Zhang XX, Zhu XQ. 2018. Prevalence and genotypes of Enterocytozoon bieneusi in pigs in southern China. Infection, Genetics and Evolution, 66, 52-56.

Cite this article as: Zhou H-H, Zheng X-L, Ma T-M, Qi M, Zhou J-G, Liu H-J, Lu G \& Zhao W. 2020. Molecular detection of Enterocytozoon bieneusi in farm-raised pigs in Hainan Province, China: infection rates, genotype distributions, and zoonotic potential. Parasite 27, 12. 
Reviews, articles and short notes may be submitted. Fields include, but are not limited to: general, medical and veterinary parasitology; morphology, including ultrastructure; parasite systematics, including entomology, acarology, helminthology and protistology, and molecular analyses; molecular biology and biochemistry; immunology of parasitic diseases; host-parasite relationships; ecology and life history of parasites; epidemiology; therapeutics; new diagnostic tools.

All papers in Parasite are published in English. Manuscripts should have a broad interest and must not have been published or submitted elsewhere. No limit is imposed on the length of manuscripts.

Parasite (open-access) continues Parasite (print and online editions, 1994-2012) and Annales de Parasitologie Humaine et Comparée (1923-1993) and is the official journal of the Société Française de Parasitologie. 